\title{
weekEndo cresce ancora
}

\author{
Maura Arosio ${ }^{1} \cdot$ Francesco Trimarchi $^{2}$
}

Accettato: 8 novembre 2021 / Pubblicato online: 21 gennaio 2022

(c) The Author(s), under exclusive licence to Springer Nature Switzerland AG 2022

La rubrica weekEndo avvia la propria storia il 14 maggio 2015 con la pubblicazione online sul sito SIE di un quesito molto semplice e diretto relativo all'effetto iperglicemizzante del Nilotinib (http://www.societaitalianadiendocrinologia. it/public/pdf/QUIZ1.pdf). La spiegazione, offerta ai solutori del quiz, era sì articolata ma molto concisa e sufficiente a fornire una corretta chiave di lettura della risposta corretta. Fu Nicola Sicolo, coadiuvato da un comitato ristretto, ad avviare la pubblicazione della rubrica che, in pochi mesi, assunse una periodicità regolare, talora settimanale, in genere quindicinale, ottenendo un crescente gradimento da parte dei Soci e dei numerosi solutori, il cui numero si attesta ormai sulle diverse centinaia.

Dopo una prima fase sperimentale e la regolare pubblicazione, il coordinamento della rubrica fu assunto da Concetto Regalbuto, cui si deve il definitivo consolidamento della rubrica e la responsabilità, nell'ambito del Comitato di redazione de l'Endocrinologo, di selezionare i quiz i cui temi, in base al numero di risposte non corrette, hanno richiesto un ulteriore approfondimento in brevi articoli pubblicati a stampa sotto la testatina della rubrica. Dal 2022 Concetto Regalbuto lascia volontariamente il coordinamento della rubrica che ha guidato, con la fruttuosa collaborazione di Angelo Cignarelli, Alberto Falorni, Federico Gatto, Paola Loli e Giorgio Napolitano, che, per le normali dinamiche redazionali, lasciano il comitato di redazione con i ringraziamenti per il loro impegno e il loro contributo. Il ringraziamento deve essere esteso a tutti gli autori che hanno redatto i quiz e le "spiegazioni" alla scelta della risposta giusta e che, dopo una fase in cui i quiz erano anonimi, appaiono regolarmente come autori del quiz e della relativa spiegazione.

La Presidente Annamaria Colao considera strategica la rubrica per la politica culturale-formativa di SIE e ne in-

$凶$ F. Trimarchi

francesco.trimarchi@unime.it

1 Dipartimento di Scienze Cliniche e di Comunità, Università degli Studi di Milano, Milano, Italia

2 Dipartimento di Medicina Clinica e Sperimentale, Università di Messina, Messina, Italia coraggia una stabile e articolata organizzazione redazionale, anche in base al successo e l'apprezzamento di questo "gioco culturale" costantemente sostenuto dai Presidenti che si sono succeduti negli anni, ultimo Francesco Giorgino. È prossima la disseminazione del quiz per mezzo di una App che lo renda ancora più immediatamente fruibile in tempo reale.

Auspicando che la periodicità quindicinale possa divenire settimanale, come prefigurato dal titolo della rubrica, e che la rivista possa ospitarne un commento in ogni numero, il coordinamento della rubrica è stato assunto da Maura Arosio, che fa parte del comitato di redazione dalla fondazione della rubrica con Maria Rosaria Ambrosio (coordinatore associato) e a due vice-coordinatori, entrambi caporedattori coordinatori della rivista (Alessandro Peri e Laura Sciacca) con il fattivo contributo di un Comitato scientifico costituito da alcuni componenti il Comitato di Redazione della Rivista e da altri giovani Colleghi, oltre che dal referente di EnGioI, Daniele Santi e dal Coordinatore della Commissione Didattica SIE, Paolo Moghetti, come riportato nella Fig. 1. Il nuovo gruppo di Coordinamento avrà il non facile compito di mantenere e implementare l'alto grado di interesse tra i Soci raggiunti durante la gestione di Nicola Sicolo e Concetto Regalbuto. Tutti i campi dell'Endocrinologia, Diabetologia e Malattie Metaboliche, Andrologia e Scienza dell'Alimentazione dovranno essere toccati con quiz preparati da colleghi esperti nell'argomento, che siano di stimolo per un costante aggiornamento e, a tale scopo, viene sollecitato l'impegno di tutti i Soci.

Nota della casa editrice Springer Nature rimane neutrale in riguardo alle rivendicazioni giurisdizionali nelle mappe pubblicate e nelle affiliazioni istituzionali. 


\begin{tabular}{|c|c|}
\hline \multicolumn{2}{|l|}{ Coordinamento } \\
\hline Maura Arosio, Milano & Coordinatore \\
\hline Maria Rosaria Ambrosio, Ferrara & Coordinatore associato \\
\hline Alessandro Peri (EB) & Vice coordinatore \\
\hline Laura Sciacca (EB) & Vice coordinatore \\
\hline \multicolumn{2}{|l|}{ Comitato scientifico } \\
\hline Giorgio Arnaldi, Ancona & Surrene \\
\hline Tommaso Aversa, Messina & Endocrinologia pediatrica \\
\hline Antonio Bianchi, Roma & Ipotalamo ipofisi \\
\hline Maria Vittoria Davi', Verona & Endocrinologia oncologica (e NET) \\
\hline Silvia Grottoli (EB) Torino & Neuroendocrinologia \\
\hline Flavia Magri , Pavia & Tiroide \\
\hline Gherardo Mazziotti (EB) Milano & Osso e paratiroidi \\
\hline Teresa Mezza (EB) Roma & Diabete e Metabolismo \\
\hline Paolo Moghetti, Verona & Coordinatore Commissione Didattica SIE \\
\hline Mirko Parasiliti Caprino, Torino & Endocrinologia cardiovascolare \\
\hline Daniele Santi (EB) Modena & Coordinatore EnGiol \\
\hline Andrea Tumminia, Catania & Metabolismo e scienza dell'alimentazione \\
\hline Massimo Scacchi Milano & Obesità e dislipidemie \\
\hline Maria Chiara Zatelli (EB) Ferrara & Malattie rare \\
\hline Marco Zavattaro. Torino & Andrologia \\
\hline
\end{tabular}

Fig. 1 Coordinamento e Comitato Scientifico weekEndo. EB, comitato di redazione l'Endocrinologo. 